\title{
A CASE OF RECURRENT APHTHOUS UVEITIS WITH ASSOCIATED ULCUS VULVAE ACUTUM (LIPSCHÜTZ)*
}

BY

A. RUGG-GunN

LONDON

ISOLATED cases of this rare and interesting disease have been described under a variety of names from time to time. Franceschetti has traced one description as far back as 1772; occasional cases were reported towards the end of the nineteenth century; but it is only within the past fifteen years or so that it has been definitely recognised as a distinct clinical entity. Typically, the symptoms fall into three main groups: (1) periodically recurring uveitis; (2) skin eruptions of various types, e.g., papular or papulopustular, i.e., a papule resembling that of secondary syphilis with a pustular centre; recurring erythema nodosum-tender, rose-pink nodules in the subcutaneous tissue, some of which may be haemorrhagic; and transient subcutaneous nodules not involving the skin and associated with fever; (3) aphthous ulcers in the mouth and on the genitals, genital herpes, and, more rarely, the ulcus vulvae acutum of Lipschütz. In the male these aphthae develop on the glans, prepuce and scrotum, as well as in the mouth.

The disease affects young persons of both sexes, usually in the decade 20 to 30 , and evolves slowly, by means of relapses, over a number of years. The three groups of symptoms constituting the syndrome are not necessarily present at the same time, and, as a rule, the eye symptoms appear to develop rather late. Once attacked, however, the eyes steadily deteriorate and progress, with remissions and relapses, towards blindness. One peculiarity of the disease as it affects the eyes, which is illustrated in the following description, is an alternating incidence of relapse from one eye to the other, suggesting an allergic mechanism.

\section{Report of a case}

G. M., female, aged 22 years, married, attended my clinic at the Central Middlesex.County Hospital on December 5, 1944, complaining of pain and blurred vision in the left eye of three days' duration. Three teeth, which were said to have been septic, had been extracted five weeks previously. The patient neither smokes nor drinks, has always had good health, but said that she had lost weight recently. Her weight was then 7 st., 6 lbs. During

* Received for publication, April 9, 1947. 
her husband's absence in the Army she had been employed as a clerk in a Government department. She is an only child. Her parents are alive and healthy, except that her mother is said to have a weak heart. There is no personal or family history of aphthae. R.V. $=6 / 9 ; \mathrm{L} . \mathrm{V} .=6 / 36$ partly. The condition of the left eye was a mild acute iritis; no posterior synechiae, but the pupil dilated unevenly under atropine; there was colloid in the A.C., but no K.P. The blood W.R. was negative. A few days later the left macula was oedematous and there appeared a small patch of cloudy exudate at the periphery below. There were now large particles and much colloid in the A.C. and two or three spots of fine K.P.

By February 6, 1945, the media were much clearer. On February 20, however, the left vitreous was more hazy. There was no fresh K.P. but a circum-corneal flush was present for the first time and a new area of oedema just above the macula, with one small round haemorrhage. Mantoux reaction one inch diameter. The vitreous gradually cleared, but was again turbid on March 27. A report from the Ear, Nose and Throat Department said that there was no nasal obstruction or discharge; some chronic inflammation of both tonsils; X-rays of sinuses clear.

During the next month, apart from the appearance of a pigmented spot in the left macula, the eye continued to improve until April 17, when Cloquet's canal became hazy. The fundal periphery, however, remained clear. The blood sedimentation rate was: 1st hour $/ 14 \mathrm{~mm}$. (normal 5-8); 2nd hour $/ 31 \mathrm{~mm}$. (normal 8-14). She was examined by Dr. Joules and the chest X-rayed. $\mathrm{A}$ few adhesions were reported at both right and left bases and $a$ calcified gland at the left hilum, but there was no evidence of pulmonary tuberculosis clinically or by X-ray. All the other systems were normal.

Early in July the right eye became inflamed for the first time. R.V. had fallen to $6 / 24$. The vitreous was dusty, with cells in the A.C., and a small area of diffuse choroiditis near the macula, which was not visible with red-free (u.v.) light. The vitreous haze already noted in the left eye was still there, also the spot of pigment at the macula. A fortnight later the right macular lesion was smaller, but there was an increased shimmering reflex in this area. Somewhat later, on July 31 , the haze in the left vitreous greatly increased. The right vitreous began to improve, but the enhanced macular reflex remained. The left vitreous became gradually more turbid. Several lesions could be seen in both fundi, but there was no more K.P.

At this stage the patient was very ill and felt giddy. She complained of night sweats, and the chest and abdomen were $\mathrm{X}$-rayed, 
with negative results. Cerebro-spinal fluid W.R. negative. Blood : Hb. 65 per cent.; W.B.C. 13,100 per c.c.; polymorphs 55 per cent., lymphocytes 36 per cent.; monocytes 5 per cent.; eosinophils 3 per cent.; basophils 1 per cent. Urine, acid; sp.gr. 1022; nothing abnormal found. Two teeth were extracted and this was followed by a rise in temperature, $101^{\circ} \cdot 2 \mathrm{~F}$. During the early part of September the evening $\mathrm{T}^{\circ}$ regularly rose to $100^{\circ}$, and during the latter half of the same month to $103^{\circ} \mathrm{F}$. She was admitted to hospital on August 23 and treated with sulphonamides. A total of $40 \mathrm{grms}$. sulphamezathine and $35 \mathrm{grms}$. of sulphathiazole was administered without any effect.

On October 16 the left eye suffered another acute relapse. Microscopic examination of the cerebro-spinal fluid revealed a few leucocytes and occasional hyaline casts. B.S.R. $12 \mathrm{~mm}$./1st hour. A fortnight later the media of the right eye, which had been steadily clearing, suddenly became dull. . The right eye, however, after this temporary lapse, again began to clear, but a new lesion (choroidal-not visible with red-free light) appeared in the macular region of the left fundus. During November both eyes became noticeably clearer, until on December 11 the patient reported that the right eye had been inflamed and "black" for a week. This proved the most severe of the recurrences in the right eye. There were two spots of fresh K.P. and the media were very turbid. The disc was obscured by exudate and vitreous haze. A large grey area, apparently of exudate, was present in the region of the macula. Some new, minute spots were seen in the left macula. B.S.R. $8 \mathrm{~mm} . / 1$ st hour. The media of the right eye soon began to clear, but early in January, 1946, the patient again developed a temperature and complained of aching of the left upper jaw. Five teeth were extracted, happily without any local reaction or rise of temperature.

Some time towards the end of November or beginning of December, 1945, apparently about the time of the last severe relapse in the right eye, the patient had a rigor, which, she said, lasted on and off for three days, and was sufficiently severe to cause chattering of the teeth. Rigors recurred at intervals until April, 1946, altogether three or four. During these the patient remained at home and was not seen by me or by any of the physicians. Towards the end of January, 1946, she was confined to bed at home on account of pain in the left side of the chest. This was diagnosed as influenza. When she reported at my clinic on January 29 she was re-examined by a physician who found no evidence of pleurisy. Her temperature during this period was said to have risen to $102^{\circ} \mathrm{F}$. This illness ended with a recurrence, accompanied by circum-corneal injection, in the left eye. B.S.R. 
$25 \mathrm{~mm}$./1st hour. When I saw her on February 26 the media of both eyes, particularly the right, were clearing. R.V. $<6 / 60$; L.V. $=6 / 60$.

On this date (February 26, 1946) the patient reported for the first time a small vulval sore, situated on the left labium, which

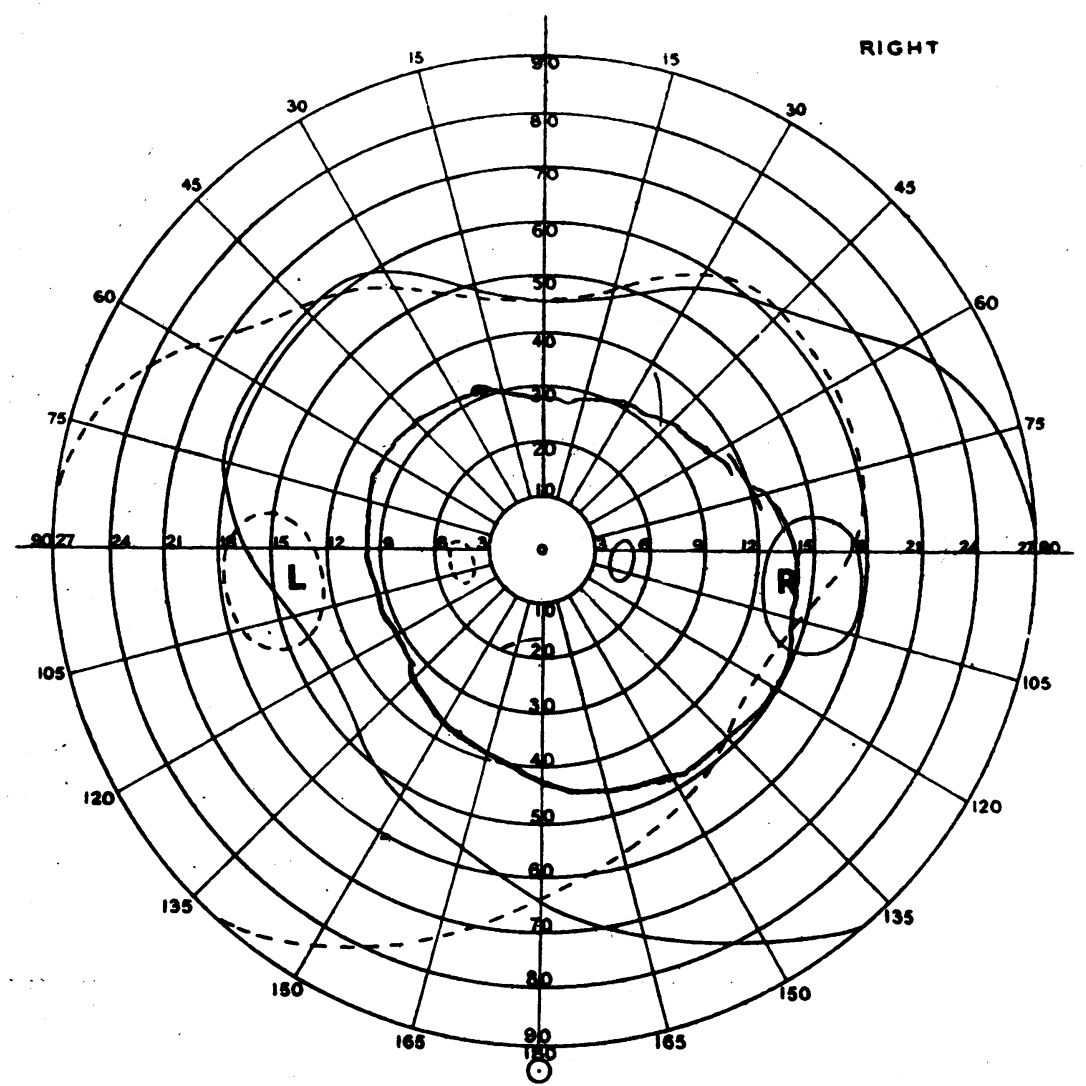

FIG. 1 .

This chart, although taken in January, 1947, is representative of a series taken of the right eye during 1946 . The object was a $3 \mathrm{~mm}$. white disk. It represents the peripheral limits of the field only, as it was found impossible to plot the irregular distribution of patches and gradients of vision and blindness which constitute the actual field.

was painful for five days but was now healed. At the same time there were small ulcers in the mouth. There were three or four of these, one on the left edge of the tongue and the others inside the cheek and inside the lower lip. The ulcers were roughly oval in shape, clean, shallow, and with sharply defined edges, approximately 2-5 $\mathrm{mm}$. in length. A fortnight later the patient had a 
severe uterine haemorrhage with clots and was pale but not collapsed. She was readmitted to hospital under a gynaecologist.

On examination no pelvic inflammation was found. By this time the patient's husband had been demobilised, but the patient denied the possibility of pregnancy and miscarriage. Subsequent events, however, suggest that this was the explanation. There was no vulval ulceration at this time, but the gynaecologist reported "a "peculiar condition of the vaginal mucosa." The vagina was granular and tender, and the mucosa of the vault hard, rough and irregular. B.S.R. $15 \mathrm{~mm} . / 1 \mathrm{st}$ hour. After a few days in hospital she was discharged.

She was readmitted on April 3 for further investigation. She now complained of tiredness, night sweats, loss of weight, and a recurrence in the right eye. There had been a recent rigor. The right eye had one posterior synechia and the vitreous was very turbid. Throughout her stay the evening temperature was $99^{\circ} .2$. The right tonsillar gland was slightly enlarged, but the tonsils were small and were reported normal. Clinical examination of the chest and X-ray examination negative. Hb. 100 per cent.; W.B.C. 9,800 ; reds $4,250,000$; platelets 425,000 approx; bleeding-time two min.; clotting-time three min., 20 sec. A catheter specimen of urine showed occasional leucocytes and reds; no organisms in a Gram film, and culture sterile. Throat swab-no haemolytic streptococci. Serum failed to agglutinate Brucella abortus. There were 31 units antistreptolysin "O " present per c.c., which is within normal limits. Mantoux $1: 10,000++$. Capillary resistance test negative. A complete clinical examination was made with negative results, but five teeth were regarded as suspicious and were extracted. The socket of one of these was septic for some time after extraction. There was one small ulcer present on the tip of the tongue.

On May 6, 1946, the left eye flared up again. The pupil did not dilate and there was yellowish discolouration of the iris, with much colloid in the A.C. and corneal epithelial oedema. The tension of the left eye was soft. Owing to the corneal oedema and turbidity of the vitreous, the pupillary red reflex was poor, but a yellowish grey mass could be detected in the upper nasal region of the posterior vitreous. The right eye had not changed; there were still massive vitreous opacities and also the lesion at the macula already noted. For some weeks both eyes remained more or less in this condition, except that the red reflex improved somewhat in the left eye, although the mass in the vitreous was still present.

The patient was admitted to the Western Ophthalmic Hospital nn July 12. The Mantoux reaction was over three inches in dia- 
meter on the third day, and it was decided to give non-specific protein therapy. Two intra-muscular injections of milk (10 c.c. each) were followed by four intravenous TAB injections, the initial dose being 20 million B. Typhosus, 10 million B. Paratyph. A, and 10 million B. Para B. The right eye cleared very considerably and even the left eye began to show a brighter red reflex surrounding the exudation in the vitreous. This seemed to me the first response to treatment so far attained. Further, the fairly regular alternation of individual relapses, first in one eye, followed by an improvement, next in the other, had suggested to me for some time that there must be an allergic mechanism present, i.e., an attack in one eye sensitising the uvea of the other and inducing a recurrence, which in turn became the excitor, and so on, in cyclic fashion. As I still suspected an underlying tuberculous infection I decided to attempt desensitisation by means of tuberculin injections. Accordingly, the patient was given weekly subcutaneous injections of old tuberculin, beginning with $1 / 1,000,000$ mgrms., and very slightly and gradually increasing the dose. The improvement continued in both eyes. The right vitreous became very clear, apart from one or two moderately large opacities, and even the left seemed to be clearing satisfactorily, although the pupil remained irregular, the tension somewhat soft, and the A.C. shallow. The patient's general health had improved. The B.P. registered 120/60. About this time the patient announced that she was pregnant and this was confirmed by Mr. MacVine.

On October 16 the patient reported that she had noticed vulval ulceration for the past two months, and $\mathrm{Mr}$. MacVine reported a small, rather painful ulcer situated on the left labium, for which he prescribed a 2 per cent. gentian violet paint. Within a month two ulcers developed at the same site and a contact ulcer, opposita the first, on the right side. The contact ulcer, however, rapidly healed and has not recurred. The original ulcer of the left pair grew rapidly, and was indurated, with raised edges, and a base composed of a thin layer of firm, slightly nodular, pale granulations, covered by a thin, light-coloured film. Mr. MacVine performed a biopsy, removing a wedge through the base of the ulcer, and inserting four catgut sutures. Dr. W. Pagel reported on the section as follows: "the ulcer goes fairly deep into the submucosa, its floor being formed almost completely by polymorphs. There is no clue as to the aetiology in the section examined. In the periphery of the ulcer the cells are chiefly. lymphocytic: there are also some eosinophils and plasma cells. We are preparing a Ziehl-Nielsen section but will not report unless acid-fast elements are found." B.P. 136/70 ; B.S.R. $16 \mathrm{~mm} . / 1 \mathrm{st}$ 
hour. Penicillin was administered locally and systemically, a total of 735,000 units, with no response. Later, the vulval ulcer was treated with a concentrated solution of urea. At the same time small ulcers, some with a sloughy base, appeared on the tongue and lips, especially on the right edge of the tongue. Culture from these yielded a few colonies of streptococcus viridans.

Just before treatment with penicillin, a rhinological report described some thickening of the lining membrane of both antra, particularly the left, and.pronounced the frontal and posterior sinuses, and the posterior ethmoidal cells as normal. The hands were again examined by $\mathrm{X}$-rays for cystic changes and were found normal. Analysis of the cerebro-spinal fluid gave protein 20 mgrms. per cent.; no excess of globulin ; chlorides $720 \mathrm{mgrms}$. per cent.; W.B.C. 1 per cent. c.mm.; R.B.C. 250 per c.mm. The pressure was normal, and the Lange curve 0012110000. The W.R. was negative, and the complement fixation test (blood-serum) for gonorrhoea also negative.

It had now become clear that the diagnosis of this condition was the syndrome which is, perhaps, best described by the term recurrent aphthous uveitis, and that the vulval ulcer was the ulcus vulvae acutum of Lipschütz. Both eyes were still fairly quiet, the vulval ulcer very painful, and the apththae in the mouth troublesome. On December 7, however, the left eye suddenly flared up and became quite blind. A necrotic, apparently encapsuled exudation occupied the whole of the posterior vitreous, giving rise to the amaurotic cat's eye reflex of Beer, and pressed forwards the lens, which remained clear, so that the A.C. was nearly obliterated and the pupil semi-dilated and fixed. A large emergent conjunctival vessel developed on the temporal side in the position of the ciliary body. The right fundus, on examination with red-free (u.v.) light, showed pale spots and streaks in the macular area, evidence of early micro-cystic degeneration of the retina. The optic disc looked very yellow-the patient is a blonde. There was a moderate amount of aqueous flare in the right A.C. and a large amount in the left. The larger of the vulval ulcers showed signs of healing, forming a waist across its centre. $\mathbf{\Lambda}$ further recurrence in the right eye was noted on February 24 and the media again became very turbid for some days. There were no ulcers in the mouth-the last was on February 18. Just before this last attack in the right eye the vision with correction $(0.5$ cyl. axis $\left.150^{\circ}\right)=6 / 36$.

At first the foetal presentation was a breech, but towards the end of pregnancy it corrected itself. Mr. MacVine performed a Caesarian operation on March 6 and extracted a female child, weighing just over $5 \mathrm{lbs}$. but otherwise normal. During the night 
Ulcus vulvae acutum of Lipschütz. When the drawing was made the ulcer had begun to heal. Its maximum dimensions were $23 \mathrm{~mm}$. by $20 \mathrm{~mm}$. In the figure the left thigh is flexed and abducted in order to expose the ulcer fully.

FIG. 2. 
of March 9 the patient had a severe rigor, accompanied by a temperature of $105^{\circ} .2 \mathrm{~F}$. , and two more next day. Afterwards convalescence proceeded normally and the patient was discharged from hospital on March 26. A very mild and transient relapse occurred in the right eye shortly after the last rigor, and on March 19 the left eye became painful, interfering with sleep for about three days. On March 18 both vulval ulcers had completely healed with a normal and perfectly healthy scar. The patient felt and appeared to be remarkably, well. A curious and perhaps significant point in connection with the vulval ulcers is that from first to

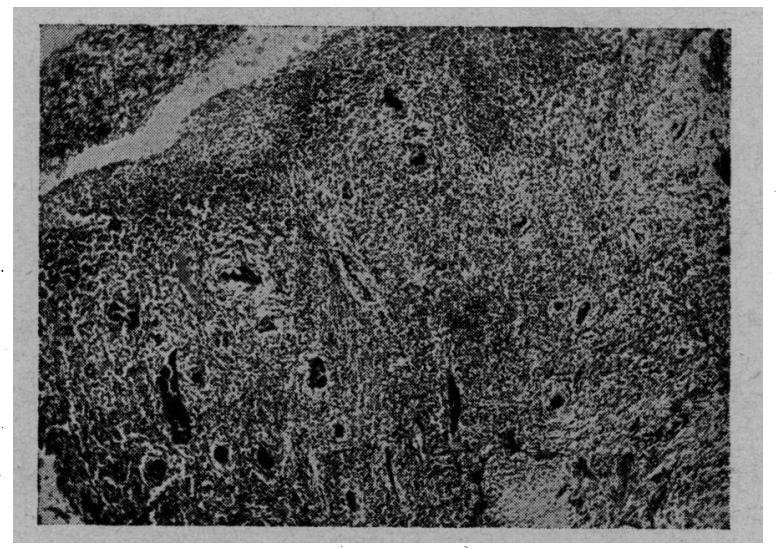

last, covering eight or nine months, there was no enlargement of the inguinal glands. Moreover, the second group of symptoms characteristic of this syndrome, viz., skin lesions and eruptions, has been entirely absent. The patient has a fine, healthy skin, above the average, and at no time has there been a trace of papules, spots or other lesion.

\section{Comments}

I have given the history of this case in detail and at length, perhaps in too much detail and at too great length, but in describing an obscure and apparently rare disease - one which, so far as I know, has not been described before in British ophthalmological literature - with an unknown aetiology, it is impossible to foresee which of its manifestations will prove to be relevant in the light 


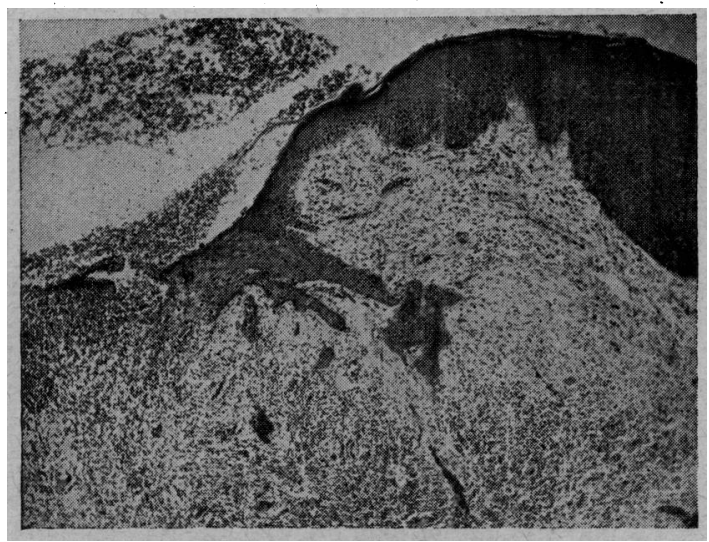

Fig. 4.

The same as Fig. 3. Margin of the ulcer showing the epithelialized edge.

of fuller knowledge. During the long course of the disease my own attitude to its probable aetiology passed through three stages: (1) the focal sepsis phase; (2) the tuberculous phase; (3) the viral phase. The initial attack of iritis, which ushered in the disease, appeared then to be the ordinary iritis or iridocyclitis that is not uncommon in young adults. A history of tooth extraction a short time previously seemed to confirm this assumption, and further investigations were planned on this hypothesis, which was not shaken by finding a rather high blood sedimentation rate. Before

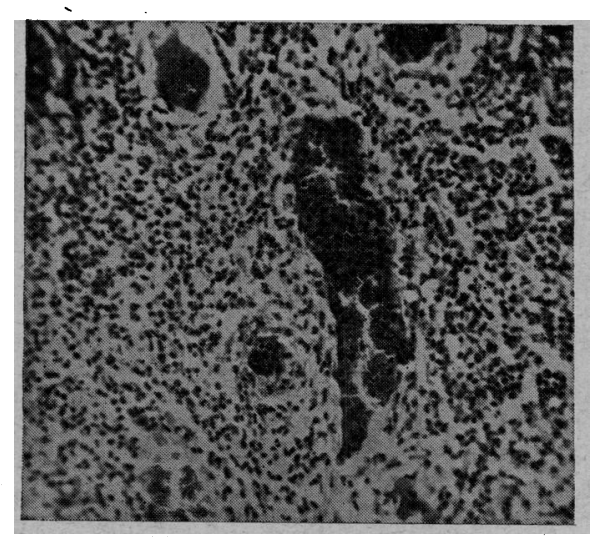

FIG. 5 .

The same as Fig. 3. High power. 
long, however, the loss of weight, night sweats and pyrexia, associated with a pronounced Mantoux reaction and high B.S.R., especially after the second eye had become involved, led me to suspect, even after clinical examination and X-rays of the chest had proved negative, an occult tuberculous infection. As the disease progressed, the apparent alternation of attacks from one eye to the other, lent colour to this suspicion, as it is generally agreed that sensitisation of tissues by tuberculous toxins explains some of its phenomena. The following table shows the dates of incidence from the beginning up to the time of writing :

TABLE I

\begin{tabular}{l|r|r|r|r|r|r|r|r|r|r|r|r|r}
\hline 1944 & \multicolumn{5}{|c|}{1945} & \multicolumn{3}{|c|}{1946} & \multicolumn{2}{|c|}{1947} \\
\hline L. 2/12 & $20 / 2$ & - & $31 / 7$ & $16 / 10$ & - & - & $29 / 1$ & - & $7 / 5$ & $7 / 12$ & - & - & $19 / 3$ \\
R. & - & $5 / 7$ & - & - & $30 / 10$ & $11 / 12$ & - & $3 / 4$ & - & - & $24 / 2$ & $10 / 3$ & - \\
\hline
\end{tabular}

Altogether there have been fourteen attacks, eight in the leit eye and six in the right. The series does not show exact alternation, but it is probably sufficiently close to conform with the theory of sensitisation. It may be remarked too that certain observers, e.g., Stahli (1922), Urbanek (1934), and Meller (1934), who have reported cases of this disease, have advocated a tuberculous aetiology.

For over a year there were no aphthae in the mouth, nor as far as I knew, on the genitals. When buccal aphthae did appear, not sufficient attention was paid to them as the physician who was then in charge regarded them as dyspeptic ulcers. It must be remembered, also, that the teeth gave trouble on several occasions and some were extracted. This was a mistake of which I was not cognisant at the time, but, fortunately, there is no evidence that it provoked a focal reaction. The buccal ulcers, however, were at first more or less unconsciously associated with the condition of the teeth.

A remarkable feature of the relapses was their sudden onset and relatively short duration. Without any warning, as it were, an eye became affected acutely overnight, reaching its maximum intensity all at once, and then declining in a day or two. This also must be regarded as compatible with an allergic reaction, and I think it must be accepted that allergy does play a part in the symptomatology of this disease, although, as Franceschetti remarks, of a secondary character. Hypopyon, a characteristic feature of early descriptions, apparently of a transient character, 
appearing and reappearing many times in the course of a single day, was never present in this case.

It was not until vulval ulcers appeared, some eighteen months after the initial attack in the left eye-apparently one or perhaps two vulval sores developed six months earlier, but were not reported by the patient until later-that the true nature of the condition became evident. The striking and very intractable ulcer, which developed on the labium majus, and proved absolutely resistant to every form of local treatment applied, was then recognised as identical with that described by Lipschütz in 1913 under the name ulcus vulvae acutum. Complete indifference to treatment, indeed, is the predominant feature of this disease. At one time non-specific protein therapy seemed to produce a favourable result. The patient herself was convinced of this, but the explanation of the apparent improvement then observed may quite well be, and probablyt is, that on this occasion the treatment coincided by chance with the beginning of a period of remission.

While Weekers and Reginster, in their second paper (1938), were the first to stress the independent nature of this disease, the credit for its complete elucidation belongs, in the first place, to Touraine (1941) and to some extent to Dascalopoulos (1932)l'uveite récidivante aphteuse-and, secondly, to Hulusi Bêhçet (1937) and to Franceschetti and Valerio (1939), who first advocated the theory of a viral aetiology. In support of this theory Franceschetti, Valerio and Babel (1946) state that recurrent aphthous uveitis has some resemblance to periodic ophthalmia in horses, which, however, has not been certainly proved to be 1 viral infection, and also to bovine aphthous fever, which is caused by a known virus transmissible to man.

It is not improbable that the pathogenic agents known as filtrable vira play a more important part in the causation of intraocular infections, e.g., sympathetic ophthalmitis, also a disease of the young, associated with injury and resistant to treatment, than is commonly supposed. Vira vary in size; the largest are almost as big as the smallest bacteria; the smallest approach the size of large molecules. According to one school of thought the virus is a living organism; according to another it is an enzyme. Vira, however, exhibit the phenomenon of variation, which is a characteristic of living matter but not of enzymes. Essentially they are cell rather than body infections, as they appear to lack the enzymes necessary for independent growth. This peculiarity may explain their immunity from the effect of drugs. In other words, they are intra-cellular parasites, and in many diseases "inclusion bodies" are seen in the infected cells, e.g., the 
Guarneri bodies of smallpox ; Negri bodies of rabies ; ProwazekHalberstaedter inclusions of trachoma, etc. They attack the cells of all three germinal layers, with the exception of muscle cells, and tend to infect young, healthy cells and young, healthy individuals rather than old, unhealthy ones. Rivers has explained that the part played by injury in causing viral lesions is due to the presence of young cells participating in the process of repair. For the same reason, embryonic cells are the best nutrient medium for their cultivation. The viral diseases constitute a large group of diverse conditions, such as measles, poliomyelitis, yellow fever, coryza, Rous' fowl sarcoma, etc. As a rule, one attack confers a life-long immunity, but influenza, the common cold, and herpes simplex are exceptions. They do not respond to sulphonamides, penicillin, or to any other known agent. Professor Bêhçet and his colleague Professor Braun, of the University of Istanbul, claim to have demonstrated inclusion bodies, or rather, the " elementary bodies," of which the cell inclusions are composed, in smears from the mouth and genital lesions of two cases. The smears were stained by the Giemsa and Herzeberg methods and formations resembling the elementary bodies of viral disease were found with each recurrence. Three patients, who also had aphthous ulcers of the mouth or genitals, were used as controls, and smears examined. Bêhçet and Braun, however, failed to find these bodies in the material from the controls. When stained with Giemsa, the bodies are approximately the same size as Guarneri bodies, i.e., extremely small and round, reddish purple in colour, partly intra-cellular and partly extra-cellular. The same bodies were found after staining with Victoria-blue by the Herzeberg method.

These findings were observed on three different occasions and experiments were devised to determine whether they were artefacts or not. For example, smears dried in air and treated with alcoholether in order to extract any lipoids present, then stained with Giemsa and Herzeberg, showed abundance of these bodies. Also when treated with 3 per cent. acetic acid solution for fifteen minutes and stained, the picture was the same. After treatment with 3 per cent. sodium hydroxide solution for fifteen minutes, however, these bodies were no longer found. Smears taken from the vulval ulcer in my case by Dr. J. D. A. Gray and examined by Dr. F. O. MacCallum of the Wellcome Bureau of Scientific Research, proved negative. It should be noted, however, that when these smears were taken the ulcer was healing rapidly and for the present the negative result must be regarded as inconclusive.

Finally, attention should be drawn to one or two unusual features in my case. First, the fact of pregnancy. It is difficuit even now to judge whether pregnancy and the metabolic changes 
associated with it affected the course of the disease one way or the other. It is true that about the sixth month of pregnancy the left eye suffered its most severe attack and became blind, and that for the greater part of the period the vulval ulcer, not only showed no signs of healing, but became gradually worse, yet the general impression in my mind was that on the whole the effect was favourable. The ulcer began to heal during the last month or two of gestation and healed very rapidly after delivery. It may be recalled that there was a probable earlier miscarriage. Aphthae appeared from time to time during pregnancy. It is interesting to note here an observation by a colleague, viz., that his wife who is subject to buccal aphthae, without other symptoms, during her pregnancies has always been free. The pregnancy of my patient was attended throughout by no untoward symptoms or discomfort.

Secondly, at one stage, lasting about five months, there occurred three or four severe and inexplicable rigors and again three days after delivery three rigors, apparently unconnected with the Caesarian section, for there was no evidence of uterine infection, followed one another within a period of about twelve hours. Otherwise, convalescence was uneventful.

Attention has already been called to the entire absence of skin symptoms; to the absence of leucocytosis; and to the absence of response to penicillin, sulphonamides, and other forms of treatment. Taken as a whole, the evidence, some positive and some negative, seems to point to infection by a virus as the most probable explanation of the nature of this disease.

\section{Summary}

The history of a case of recurrent aphthous uveitis is described over a period of twenty-eight months.

There were no dermatological symptoms but the ulcus vulvae acutum of Lipschütz was present.

Pregnancy occurred and went on to term.

Rigors occurred on several occasions.

Resistance to treatment is emphasised.

The hypothesis of a viral infection is discussed.

I have pleasure in expressing my thanks to Dr. Joules, the Medical Director, and to other members of the staff of the Central Middlesex County Hospital, especially Mr. MacVine, Dr. W. Pagel and Dr. J. D. A. Gray, for their invaluable help and co-operation. 


\title{
REPERENCES
}

For a full bibliography the classic paper of Franceschetti, Valerio and Babel should be consulted. The following are papers which were consulted by myself, and used in the text.

BÊçHET.-Dermat. Wochenschr., Vol. CV, p. 1152, 1937.

- Bull. Soc. franc. Derm. Syph., Vol. XLVI, p. 674, 1939.

Dascalopoulos, N.-Ann. d'Ocul., Vol. CLXIX, p. 387, 1932.

FranceschetTi, A., Valerio, M. and Babel, J.-Arch. Ophthal., Vol. XXXV, p. $5,1946$.

Lipschütz, B.-Arch.f. Dermat. u. Syph., Vol. CXIV, p. 363, 1913.

Thomas, E. W. Prosser.-Brit. Med. Jl. January 4, 1947.

Touraine, A.-Presse Méd., Vol. XLIX(I), p. 571, 1941.

URBANEK, J.-Zeitschr. f. Augenheilk., Vol. LXIX, p. 174, 1929.

- Ibidem., Vol. LXXXIII, p. 357, 1934.

WEEKERS, L. and RegINSTER, H.-Bull. Soc. belge d'Optal., Vol. LXXVI, p. 31 1938.

- Arch. d'Ophtal., Vol. II, p. 687, 1938.

Weve, H.-Arch.f. Augenheilk., Vol. XCIII, p. 14, 1923.

Whitwell. G. P. B.-Brit. Jl. Dermat, and Syph., Vol. XLVI, p. 414, 1934.

\section{FURTHER EXPERIENCE WITH AMNIOTIC MEMBRANE GRAFTS IN CAUSTIC BURNS OF THE EYE*}

\author{
BY \\ Arnold Sorsby, Joan Haythorne \\ and HOWARD REED \\ LONDON
}

IN an earlier communication (Sorsby and Symons, 1946) attention was drawn to the value of amniotic membrane grafts in the treatment of caustic burns of the eye. In a series of 30 cases (the burn being produced by lime in 22 cases; by hydrochloric acid, sodium hydroxide, and ammonia in 2 cases each; and by liquid sulphur dioxide and lead in 1 case each) no corneal or conjunctiva sequelae were noted in 27 cases. In 28 eyes in which vision was known 26 showed vision of 6/9-6/5. A method of applying the graft was described and it was indicated that early grafting gives the best results. Generally speaking the eyes were found to be very nearly white in 3 to 5 days after grafting and they were usually normal by the end of a week.

\section{1.-A further series of 28 cases}

Table I sets out the distribution and the clinical details in a further series of 28 cases. These fall into three distinct groups :

- Received for publication, May 5, 1947. 